\title{
Funktionelles Finish für edle Bleche
}

\section{Werkzeugbürsten dienen der gezielten optischen und funktionellen Optimierung von Metallblechen. Je nach Anwendung stehen dazu verschiedene Bürstentypen zur Verfügung, die durch unterschied- liche Besatzmaterialien, Borstendicken und Besatzdichten vielfältige Einsatzmöglichkeiten bieten.}

Ob die Aufwertung des Produktdesigns, die Verbesserung der Reinigungsfähigkeit oder die Vorbereitung für den nächsten Prozessschritt - es gibt vielfältige Gründe für die gezielte optische oder funktionelle Optimierung der Oberflächen von Metallblechen. Häufig wird dieses oberflächentechnische Finishing mit anwendungsspezifisch ausgelegten Werkzeugbürsten ausgeführt.

Die Spiralwalzenbürsten von Kullen-Koti kommen für ein großflächiges und hocheffizientes Oberflächen-Finish von Stahl-, Edelstahl-, Leicht- und Buntmetallblechen zum Einsatz. Die Bürsten werden in verschiedenen Ausführungen mit Vollbesatz, spiralförmig mit Steigung sowie mit Einzel- oder Doppelband angeboten. Sie arbeiten mit zum Teil hohen Drehzahlen und lassen sich sehr flexibel auf den konkreten Anwendungsfall abstimmen.

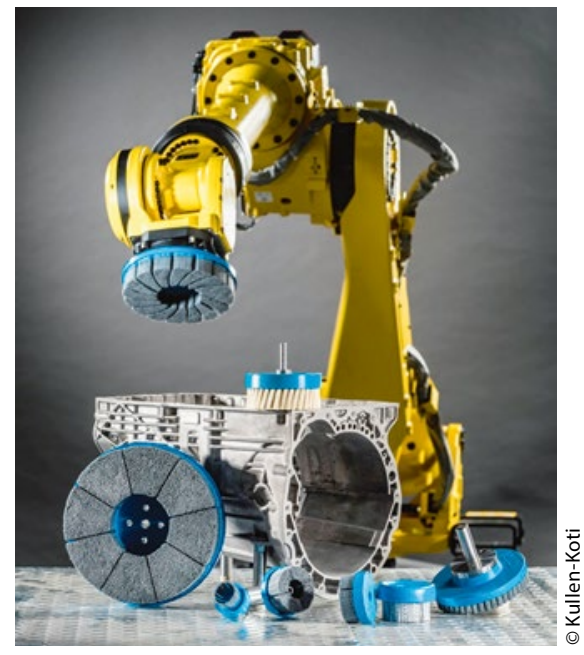

Vollautomatisierter 3D-Einsatz gegossener Tellerbüsten auf einem Roboter.
Wichtige Kriterien sind dabei nicht nur das Besatzmaterial, sondern auch der Borsten-Durchmesser und die Besatzdichte. Die Spiralwalzenbürsten sind nahezu in jeder Länge beziehungsweise Breite und fast jedem Durchmesser verfügbar. Aufgrund der Wandlungsfähigkeit dieses Bürstentyps können die Oberflächen von Blechen je nach Zielsetzung sehr fein oder sehr stark gebürstet werden.

\section{Vielfältige Einsatzmöglichkeiten}

Besonders vielseitig für das Oberflächen-Finishing von Blechen einsetzbar sind auch die gestanzten Rund- und Walzenbürsten des Bürsten-Herstellers. Durch die vielen Kombinationsmöglichkeiten von Bürstenkörper und Besatzmaterial sowie die Realisierung anwendungsspezifischer Besatzbilder eignen sie sich für eine große Bandbreite oberflächentechnischer Arbeiten, bei denen keine hohen Umdrehungszahlen gefordert sind und keine starken mechanischen Belastungen auf die Bürste einwirken.

Typische Einsatzfälle sind beispielsweise das Einölen, Mattieren, Schleifen oder Reinigen von Metallblechen. $\mathrm{Zu}$ den speziellen Vorteilen der gestanzten Rund- und Walzenbürsten gehören ihr geringes Eigengewicht und ihre flexible Einsetzbarkeit.

Ein weiteres Highlight in Sachen Oberflächen-Finishing bilden die Tellerbürsten, die sich ebenfalls sehr vielseitig auslegen lassen. Auch hier sind es hauptsächlich die Faktoren Besatzmaterial, Borstendicke und Besatzdichte, die entscheidenden Einfluss auf das Ergebnis haben.

Soll zum Beispiel eine stark abrasive Wirkung erzielt werden - wie etwa beim Aufrauen oder Entgraten - lassen sich die Tel- lerbürsten mit Polyamidborsten ausstatten, die mit unterschiedlichen Schleifmitteln wie Siliziumcarbid, Aluminiumoxid oder Diamant durchsetzt sind. Ist hingegen eine moderat schleifende, eine glättende oder polierende Wirkung gefordert, so werden die Tellerbürsten mit entsprechend weicheren Besatzmaterialen bestückt.

\section{Wirtschaftliche Lösung aus einem Guss}

Metallbearbeiter, die ein besonders effizientes Präzisionswerkzeug für das Oberflächen-Finishing ihrer Bleche suchen, finden bei Kullen-Koti die gegossene Tellerbürste. Im Gegensatz zu vielen anderen Bürsten, die bisher im Markt für die Fertigungsschritte Entgraten, Verrunden oder Glätten zu finden sind, ist der Besatz dieser Bürste untrennbar in die Trägerronde eingegossen. Dieser Verbund verleiht dem Besatz der Tellerbürste eine extrem hohe Formstabilität. Dadurch bleibt die Eintauchtiefe konstant und alle Verfahrensschritte lassen sich mit kontinuierlich hoher Genauigkeit ausführen. Gleichzeitig erreicht die gegossene Tellerbürste durch die verbesserte Formstabilität des Besatzes eine höhere Standzeit als herkömmliche Bürsten und muss seltener ausgetauscht werden und erweist sich somit als die wirtschaftlichere Lösung. // AMB: Halle 5, Stand A43

\section{Kontakt}

Kullen-Koti GmbH, Reutlingen Tel. 07121 142211, direktkontakt@kullen.de www.kullen.de, www.koti-eu.com 